Canadian

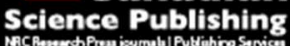

Applied Physiology, Nutrition, and Metabolism Physiologie appliquée, nutrition et métabolisme

\title{
The effect of rolling massage on the excitability of the corticospinal pathway
}

\begin{tabular}{|c|c|}
\hline Journal: & Applied Physiology, Nutrition, and Metabolism \\
\hline Manuscript ID & apnm-2017-0408.R1 \\
\hline Manuscript Type: & Article \\
\hline Date Submitted by the Author: & 20-Sep-2017 \\
\hline Complete List of Authors: & $\begin{array}{l}\text { Aboodarda, Saied; Memorial University of newfoundland, Human } \\
\text { performance and recreation; University of Calgary, Kinesiology } \\
\text { Greene, Rebecca ; Memorial University of Newfoundland } \\
\text { Philpott, Devin; Memorial University of Newfoundland, Human Kinetics and } \\
\text { Recreation } \\
\text { Jaswal, Ramandeep; University of Calgary, Kinesiology } \\
\text { Millet, Guillaume; University of Calgary, Kinesiology } \\
\text { Behm, David; Memorial University of Newfoundland, }\end{array}$ \\
\hline \multicolumn{2}{|l|}{$\begin{array}{r}\text { Is the invited manuscript for } \\
\text { consideration in a Special } \\
\text { Issue? : }\end{array}$} \\
\hline Keyword: & $\begin{array}{l}\text { massage, transcranial magnetic stimulation, afferent feedback receptors, } \\
\text { corticomotor pathway, motoneurone }\end{array}$ \\
\hline
\end{tabular}



Guillaume Y. Millet ${ }^{2}$, David G. Behm ${ }^{1}$

${ }^{1}$ School of Human Kinetics and Recreation, Memorial University of Newfoundland, St. John's, 10 Newfoundland, Canada.

$11{ }^{2}$ Human Performance Laboratory, Faculty of Kinesiology, University of Calgary, Calgary, 12 Canada

16 Corresponding author:

17 Saied Jalal Aboodarda, $\mathrm{PhD}$

18 Postdoctoral fellow, Faculty of Kinesiology

19 University of Calgary

202500 University Drive NW, Calgary, Alberta, CANADA T2N 1N4

21 saiedjalal.aboodarda@ucalgary.ca

22 http://www.ucalgary.ca/nmfl 


\section{5}

\section{Abstract}

The aim of the present study was to investigate the alterations of corticospinal excitability (motor evoked potential, MEP) and inhibition (silent period, SP) following rolling massage of the quadriceps muscles. Transcranial magnetic and femoral nerve electrical stimuli were used to elicit MEPs and compound muscle action potential (Mmax) in the vastus lateralis and vastus medialis muscles prior to and following either: i) 4 sets of 90-s rolling massage (ROLLING) or ii) rest (CONTROL). One series of neuromuscular evaluations, performed after each set of ROLLING or CONTROL, included three MEPs and one Mmax elicited every $4 \mathrm{~s}$ during $15 \mathrm{~s}$ submaximal contractions at $10 \%$ (experiment $1, \mathrm{n}=16$ ) and $50 \%$ (experiment $2, \mathrm{n}=10$ ) of maximal voluntary knee extensions (MVC). The MEP $\mathrm{Mmax}^{-1}$ ratio and electromyographic activity recorded from VL at 10\% MVC demonstrated significantly lower values during ROLLING than CONTROL $(P<0.05)$. The ROLLING did not elicit any significant changes in muscle excitability (Mmax area) and duration of TMS-induced SP recorded from any muscle or level of contraction $(\mathrm{P}>0.05)$. The findings suggest that rolling massage can modulate the central excitability of the circuitries innervating the knee extensors however, the observed effects are dependent on the background contraction intensity during which the neuromuscular measurements are recorded.

Key words: massage, transcranial magnetic stimulation, afferent feedback receptors, corticomotor pathway, motoneurone. 
47

\section{Résumé}

Le but de cette étude était d'investiguer les modifications d'excitabilité (potentiel évoqué moteur, PEM) et d'inhibition (période de silence, PS) corticospinale à la suite d'un massage par rouleau des quadriceps. La stimulation magnétique transcrânienne et la stimulation électrique du nerf fémoral ont été utilisées pour évoquer des PEMs et des potentiels d'action musculaires composés (Mmax) sur les muscles vastus lateralis et vastus medialis avant et après : i) 4 séries de 90-s de massage par rouleau (ROLLING) ou ii) une période équivalente de repos (CONTROL).

Les évaluations neuromusculaires, réalisées après chaque série de ROLLING ou CONTROL, comprenaient trois PEMs et un Mmax évoqués toutes les 4 s pendant une contraction sousmaximale à $10 \%$ (étude $1, \mathrm{n}=16$ ) et $50 \%$ (étude $2, \mathrm{n}=10$ ) de la force maximale volontaire (FMV). Le rapport MEP·Mmax ${ }^{-1}$ et l'activité électromyographique enregistrée sur VL à $10 \%$ de FMV étaient significativement plus faibles pour ROLLING que pour CONTROL $(P<0,05)$. En revanche, ROLLING n'induisait aucune modification significative de l'excitabilité du muscle (aire de Mmax) ou de la durée des PSs, quel que soit le niveau de contraction $(P>0,05)$. Ces résultats suggèrent que le massage par rouleau peut moduler l'excitabilité centrale des voies innervant les muscles extenseurs du genou. Cependant, les effets dépendent l'intensité de contraction pendant laquelle l'évaluation neuromusculaire est réalisée.

Mots-clés : massage, stimulation magnétique transcrânienne, récepteurs sensoriels, voie corticospinale, motoneurone. 


\section{Introduction}

Self myofascial release (SMFR) technique using foam roller and roller massager is used extensively in rehabilitation and athletic settings to promote soft-tissue extensibility and enhance recovery from training (for review, see Beardsley and Škarabot, 2015). Previous studies suggest that this technique may enhance range of motion (MacDonald et al. 2013; Sullivan et al. 2013; Halperin et al. 2014; Bradbury-Squire et al. 2015; Behara and Jacobson 2017), pressure pain threshold (Pearcey et al. 2015; Aboodarda et al. 2015; Cavanaugh et al. 2017) and arterial dilation and vascular plasticity (Okamoto et al. 2014). A "neurophysiological model" has been proposed to explain the influence of SMFR on the musculoskeletal functions. This model focuses on the mechanical pressure that a roller massage apparatus exerts on the mechanoreceptors, proprioceptors and pain receptors encapsulated in the fascia (for review, see Beardsley and Škarabot 2015). It has been suggested that activation of these sensory receptors alters the selfregulatory dynamics of the autonomic nervous system and consequently modifies the muscle tissue extensibility (for review, see Schleip 2003 a,b; Beardsley and Škarabot 2015).

One aspect of the SMFR technique that has not been explored is the role that it may play in the modulation of the corticospinal pathway (central) excitability throughout the activation of the afferent feedback receptors. It is well established that repeated somatosensory input (via activation of sensory receptors) can modulate the responsiveness of the motor and sensory cortical circuitries (Fourment et al. 1996; Carson et al. 1999; Ridding and Taylor, 2001; KaelinLang et al. 2002). Several studies have used transcranial magnetic stimulation (TMS) and reported an increase in the excitability of the corticomotor pathway following activation of the afferents sensory receptors with muscle and tendon vibration (Siggelkow et al. 1999; Steyvers et al. 2003; Souron et al. 2017). This contrasts with no change in corticospinal excitability with 
manual massage (Dishman and Bulbulian, 2001). Conversely, studies that used Hoffmann's reflex (H-reflex) amplitude found a reduction in excitability of the spinal motoneurone during manual massage (Morelli et a. 1991; Goldberg et al. 1992; Sullivan et al. 1991, 1993; Behm et al. 2013). However, there is no documented study that has explored the influence of the rolling massage on the responsiveness of the corticospinal pathway innervating the massaged muscle group.

Understanding the effects of rolling massage on acute corticomotor responses may reveal the mechanistic basis of the adaptations that may occur in the central nervous system following the chronic use of SMFR. Therefore, the aim of the present study was to investigate the influence of rolling massage on the corticospinal and peripheral responses of the knee extensor muscles. Based on previous massage studies, it was hypothesized that rolling massage will inhibit corticospinal excitabilities.

\section{MATERIALS and METHODS}

\section{Experiment 1}

Participants. Sixteen recreationally active male participants (height $175.5 \pm 7.8 \mathrm{~cm}$, body mass $79.4 \pm 9.1 \mathrm{~kg}$, age $27.2 \pm 8.8 \mathrm{yrs})$ volunteered for this study. Fifteen participants were determined as right-leg dominant based on the preferred leg used to kick a ball (Kovaleski et al. 1999). Individuals with neurological conditions, cardiovascular complications, or surgery or injury to the knee structures were excluded from the study. After explaining the experimental procedures, participants completed the TMS safety checklist (Rossi et al. 2011) and the Physical Activity Readiness Questionnaire-Plus form (Canadian Society for Exercise Physiology, 2011). Participants also signed a letter of informed consent prior to participating in the study. 
112 Participants were instructed to abstain from alcohol, caffeine, nicotine, and strenuous physical

113 activity for at least 24-hours prior to the experimental sessions. Ethical approval for this study

114 was granted by the Health Research Ethics Authority of the Memorial University of

115 Newfoundland (HREB \#14.118).

116

117

118

119

120

121

122

123

124

125

126

127

128

129

130

131

132

133

26

7

Research design. Participants visited the laboratory on three separate occasions separated by at least 24 hours. The first session involved familiarizing the participants with the experimental protocol and obtaining informed consent. During the next two sessions, the order of which was randomized, the participants performed one of the two intervention protocols: i) four sets of 90s rolling massage (ROLLING) applied on the quadriceps muscles or ii) time matched rest (CONTROL). A series of neuromuscular evaluations were performed before (baseline) and following each set of intervention (rolling massage or rest). All measurements and the rolling massage were performed on the right leg. participants' muscles and peripheral nerve, respectively (see below). During experimental protocol, participants were seated in a custom-built knee extension chair with the hip and knee positioned at $90^{\circ}$ (Button and Behm, 2008). In order to avoid contribution from the upper body during knee extensions, two straps were placed around the trunk and waist and participants were instructed to cross their arms across their chest. The right ankle was inserted into padded ankle cuffs attached to a strain gauge (Omega engineering Inc., LCCA 250, Don Mills, Ontario) via a non-extensible strap. The data from the strain gauge was sampled at a rate of $2,000-\mathrm{Hz}$, 
134 amplified $(\times 1000)$, digitally converted (AcqKnowledge III, Biopac Systems Inc., Holliston, MA)

135 and monitored on a computer screen.

136

137

138

139

140

141

142

143

144

145

146

147

148

149

150

151

152

153

154

155

156

Before initiation of the neuromuscular evaluations, participants performed a warm-up for the knee extensor muscles. Warm-up consisted of 2 sets of 12 submaximal isometric contractions at $50 \%$ of estimated MVC. The contractions were intermittent: 2-s contraction followed by 2-s rest. Following warm-up, two 4-s isometric knee extension MVCs were performed at baseline. Two minutes of rest was given between the MVCs. Another MVC was performed immediately after completion of the interventions (ROLLING or rest) in each experimental session. Participants were encouraged to generate maximal force output as fast as possible.

The maximal force derived from the baseline MVCs was used to calculate $10 \%$ of MVC.

This value was shown on the computer screen, which participants used as a guideline. The participants were instructed to sustain the knee extension force just above the guideline for $15 \mathrm{~s}$ during which three TMS and one peripheral nerve electrical stimulus (PNS) (Figure 1) were elicited. The time interval between the stimuli was $4 \mathrm{~s}$ and the first stimulus was delivered $2 \mathrm{~s}$ after initiation of knee extension contractions. Thus, the stimuli were delivered at 2, 6, 10 and 14 s. The sequence of TMS and PNS stimuli was randomly assigned for each participant.

Rolling massage was applied on the quadriceps muscles using a Theraband ${ }^{\circ}$ roller massager (Hygienic Corporation, Akron, $\mathrm{OH}$ ). The roller massager was $24 \mathrm{~cm}$ in length and 14 $\mathrm{cm}$ in circumference and composed of a hard rubber material with low amplitude, longitudinal grooves surrounding a plastic cylinder (Halperin et al. 2014). Rolling massage was applied over the belly of the quadriceps muscle, along the length of VL, VM and rectus femoris muscles, at a slow pace ( $2 \mathrm{~s}$ proximally and $2 \mathrm{~s}$ distally). Participants provided feedback regarding the level of 
157 perceived pain during the rolling massage and the intensity of applied force (with a depth of $\sim 1$ -

$1583 \mathrm{~cm}$ over quadriceps muscle) was adjusted accordingly to ensure a value of 7/10 on the visual analogue scale (VAS) was maintained (Halperin et al. 2014; Aboodarda et al. 2015).

Electromyography (EMG). Surface EMG activity was measured using pairs of self-adhesive Ag$\mathrm{Ag} \mathrm{Cl}$ electrodes (Kendall MediTrace foam electrodes, Chicopee, MA) positioned $2 \mathrm{~cm}$ apart

163

164 (centre to centre) on the vastus lateralis (VL) and vastus medialis (VM) muscles of the right leg in the direction of the underlying muscle fibers (Hermens et al. 1999). A ground electrode was placed on the patella bone of the same leg. In order to decrease skin resistance and ensure an inter-electrode impedance of $<5 \mathrm{k} \Omega$, the skin was shaved, abraded, and cleaned with an isopropyl alcohol swab. All EMG signals were amplified (Biopac System Inc., DA 100: analog to digital converter MP150WSW; Holliston, MA) and recorded with a sampling rate of 2,000 Hz using a commercially designed software program (AcqKnowledge III, Biopac System Inc.). EMG activity was filtered with a Blackman $-61 \mathrm{~dB}$ band-pass filter between 10-500 Hz, amplified (bipolar differential amplifier, input impedance $=2 \mathrm{M} \Omega$, common mode rejection ratio $>110 \mathrm{~dB}$ min, gain $\times 1000)$, analog-to-digitally converted (12 bit) and stored for further analysis.

Peripheral nerve stimulation. To determine the size of compound muscle action potential (Mmax), the peripheral nerves innervating the quadriceps muscle were stimulated by a single stimulus at the femoral nerve using a constant-current stimulator (DS7AH; Digitimer, Hertfordshire, UK). The surface stimulating electrodes were secured at the femoral triangle (cathode; Kendall MediTrace foam electrodes, Chicopee, MA) and between the greater trochanter and superiliac projections (anode; $9 \times 5 \mathrm{~cm}$, Dura-Stick II, Chattanooga Group, 
180 Hixson, TN). The intensity of the stimuli (70 - $340 \mathrm{~mA}$; square-wave pulse duration: $200 \mu \mathrm{s} ; 400$

181 V maximum voltage) was increased incrementally until Mmax was observed. The current

182 intensity was then increased by an additional $30 \%$ to ensure supramaximal stimulation. This

183 stimuli intensity was used for the remainder of the experimental session. Mmax was also used to

184 normalize MEP area to account for changes in peripheral neuromuscular propagation.

185

186

187

188

189

190

191

192

193

194

195 $196 \pm 14 \%$ and $47 \pm 9 \%$ of maximum stimulator output, respectively.
198

199

200

201

202

Transcranial Magnetic Stimulation. TMS induced motor evoked potential (MEP) responses of the quadriceps muscles were evoked using a single TMS pulse. During voluntary isometric knee extensions (10\% of MVC), TMS pulses were manually delivered to the motor cortex using a magnetic stimulator (Magstim 2002, The Magstim Company Ltd., Whitland, UK) and a 110-mm double-cone coil (maximum output of $1.4 \mathrm{~T}$ ) to induce a posteroanterior current. Participants wore a latex swim cap on which the coil location was drawn. The coil was positioned at the vertex marked on the scalp as the intersection of the lines drawn from nasion to inion and from tragus to tragus. TMS intensity was increased stepwise to produce a MEP amplitude of approximately $20 \%$ of VL and VM muscle Mmax during brief contractions at 10\% MVC. The group means stimulation intensities for contractions at 10 and 50\% of MVC were 61

\section{Experiment 2}

Ten recreationally active male participants (height $176.2 \pm 6.83 \mathrm{~cm}$, body mass $78.9 \pm 8.4$ $\mathrm{kg}$, age $27.6 \pm 6.6 \mathrm{yrs})$ completed the same protocol as experiment 1 , with the exception of the intensity of MVC knee extensions, which was changed to 50\%. Participants included seven participants from experiment 1 and three new participants. 
Outcome measures. MEP and Mmax areas were measured from the initial deflection of signal from baseline to the second crossing of the horizontal axis. The duration of the silent period (SP) was assessed as the interval from the MEP stimulus artifact to the return of the continuous EMG by visual inspection (Schnitzler and Benecke 1994). The MEP responses were divided by the corresponding Mmax recorded at each contraction to calculate $\mathrm{MEP} \cdot \mathrm{Mmax}^{-1}$ ratio. In order to eliminate the effect of day-to-day variations on MEP and Mmax responses, all post-intervention values (i.e. measurements following each set of rolling massage or rest) were normalized to the average of the two baseline measurements at the same contraction intensity. The background EMG (root mean square; rmsEMG) of the VL and VM were quantified over 500 ms duration prior to the point of each stimulus (TMS and PNS) at each target force. In order to evaluate the central drive during contractions, the rmsEMG values were normalized to the amplitude of Mmax recorded at each contraction. The magnitude of the baseline and post-intervention peak MVC force outputs were measured in each experimental session.

Statistical Analysis. Statistical analyses were computed using SPSS software (Version 16.0, test) were tested for all of the dependent variables. If the assumption of sphericity was violated, the corrected value for non-sphericity with Greenhouse-Geisser epsilon was reported. In order to 222 determine the effect of rolling massage on corticospinal responses of the quadriceps muscles, a two-way analysis of variance (ANOVA) with repeated measures $(2$ conditions $\times 4$ sets of interventions) was used for all variables. A two-way ANOVA with repeated measure (2 conditions $\times 2$ time points) was performed to measure the influence of the rolling massage on 
226 MVC force output. If results showed a significant main effects or interactions, Bonferroni post-

227 hoc test was used to identify differences trials. The effect size (ES) was calculated converting 228 partial eta-squared to Cohen`s d (Cohen, 1988) to provide a better understanding about the 229 magnitude of the statistical significance between different measures. According to Cohen (1988), 230 the magnitude of effect size can be classified as small $(0.2 \leq \mathrm{d}<0.5)$, medium $(0.5 \leq \mathrm{d}<0.8)$, 231 and large $(d \geq 0.8)$. This process was repeated for all variables recorded at either 10 or $50 \%$ of 232 MVC experiments. Significance was defined as $p<0.05$.

\section{Results}

235 The ROLLING did not cause any significant change in the post-intervention MVC force output 236 as well as the muscle excitability (Mmax area) at either 10 or 50\% MVC (all $\mathrm{P}>0.05$ ). 237 Additionally, no significant change was observed for the SP recorded from VL or VM during 238 contractions at either 10 or $50 \% \operatorname{MVC}(P>0.05)$. The absolute values for the 239 neurophysiological parameters are presented in Tables 1 and 2.

\section{Experiment 1}

241 MEP Area. The MEP $\mathrm{Mmax}^{-1}$ ratio recorded from VL at $10 \% \mathrm{MVCs}$ demonstrated a 242 significantly lower value (condition effect: $\mathrm{F}_{1,15}=4.75, P=0.046, d=1.12$ ) during the 243 ROLLING compared to the CONTROL session (Figure 1 and 2). No significant difference was 244 observed for the MEP·Mmax ${ }^{-1}$ recorded from VM at this contraction intensity. rmsEMG. The rmsEMG recorded from VL (normalized to Mwave) exhibited a significantly

246 lower value (condition effect: $\mathrm{F}_{1,15}=7.91, P=0.016, d=1.62$ ) following ROLLING than 247 CONTROL across the 4 sets of intervention (Figure 3). The difference between the two 
248 conditions showed similar pattern for the VM rmsEMG however the data demonstrated a trend to

249 significance $\left(\mathrm{F}_{1,15}=3.93, P=0.07, d=1.14\right)$.

250

251

252

\section{Experiment 2}

MEP Area. No significant change was observed for the VL and VM MEP·Mmax ${ }^{-1}$ ratio at this intensity $(P>0.05)$.

rmsEMG. The rmsEMG recorded from VL and VM at 50\% of MVC did not demonstrate any difference between two conditions $(P>0.05)$.

\section{Discussion}

The principal findings of the present study are: (i) ROLLING modulated (reduced) the corticospinal responses recorded from VL at $10 \%$ of MVC, (ii) no significant difference was observed in the peripheral excitability (Mmax) of the VL after the two conditions; thus these findings suggest that the observed modulations in MEP and rmsEMG responses at $10 \%$ of MVC were due to the adaptations in the central motor pathway controlling the activity of the VL. The MEP and rmsEMG recorded from VL and VM at 50\% of MVC exhibited no difference between the two conditions. Overall, the results indicate that rolling massage disfacilitates the central excitability of the circuitries innervating the massaged muscles (specifically VL). However, this effect is only evident at low level of contractions (e.g. 10\% of MVC) where minimum central drive is required to recruit the low threshold spinal motoneurones and motor units.

To best of our knowledge, this is the first study to quantify the effect of rolling massage on central and peripheral excitability of a muscle group. Indeed, several studies have examined 
270 the effect of other mechanical stimuli such as tendon vibration (Siggelkow et al. 1999; Kossev et 271 al. 1999; Steyvers et al. 2003) and manual massage (Dishman and Bulbulian, 2001) on alteration 272 of the corticomotor pathway responses. However, due to differences in the characteristics of the 273 mechanical pressure applied on the tissue, the findings of the present study can not be directly 274 compared with these studies. For instance, during the muscle and tendon vibration, a low muscle 275 vertical displacement $(0.5 \mathrm{~mm})$ and moderate to high frequency stimuli $(75-120 \mathrm{~Hz})$ were 276 applied (Siggelkow et al. 1999; Steyvers et al. 2003); whereas during ROLLING a high muscle 277 vertical pressure (with a depth of $\sim 1-3 \mathrm{~cm}$ ) and low pace of rolling massage (i.e. $2 \mathrm{~s}$ from 278 proximal to distal and $2 \mathrm{~s}$ from distal to proximal) were exerted. Nonetheless, a general comparison between the effects of the two mechanical stimuli indicates that the local vibration 280 (high frequency/low mechanical pressure) facilitated the corticospinal excitability (Siggelkow et 281 al. 1999; Kossev et al. 1999; Steyvers et al. 2003) whereas ROLLING (low frequency/high 282 mechanical pressure) resulted in the reduction of central motor responses. A possible factor 283 leading to this divergent result could be the activation of different afferent sensory receptors by 284 local vibration and ROLLING. It is well established that the low amplitude innocuous vibration 285 activates primary spindle afferents and consequently enhances the excitability of corticospinal 286 287 deep tissue massage can evoke multidimensional sensory pathways including mechanoreceptors, 288 proprioceptors and muscle nociceptors mediated by group III and IV afferents (Goldberg et al. 289 1992). Several investigators have postulated that activation of Golgi tendon organs, secondary 290 muscle spindle afferents and group III and IV pain receptors can inhibit central excitability in the 291 massaged muscles (Goldberg et al. 1992; Sullivan et al. 1991, 1993; Behm et al. 2013). 292 Interestingly, the magnitude of this inhibitory response was greater following deep tissue 
massage compared to a light massage (Goldberg et al. 1992). In the present study, the magnitude of mechanical pressure applied during ROLLING was adjusted based on the pain perception. Given that a high amplitude mechanical pressure was administered during ROLLING and participants experienced 7/10 pain sensation, it seems quite plausible to speculate that ROLLING activated a wide range of somatosensory inputs including inhibitory afferent pathways mediated by Golgi tendon organs and muscle nociceptors.

Another intriguing result of the present study was that the MEP and rmsEMG exhibited distinctive responses when neuromuscular evaluations were performed at 10 and $50 \%$ of MVC. Specifically, despite that the neuromuscular evaluations at $10 \%$ of MVC revealed a depression of VL MEP and rmsEMG responses, the two measures exhibited no difference between ROLLING and CONTROL at 50\% of MVC. The reason for this finding remains unclear; however, it can be suggested that the mechanical stimuli exerted by ROLLING had a selective inhibitory effect on the low threshold motoneurones which are contributing to low intensity contractions (10\% of MVC). In line with this explanation, Bradbury-Squire and colleagues (2015) showed a reduction in VL EMG activity during a lunge action following 5 sets of 60 -s rolling massage intervention. These investigators suggested that the lower EMG could be due to a reduction in the spinal motoneurone excitability. Caution should be taken in accepting this interpretation in the context of the present study because we did not measure spinal motoneurone responses. In fact, the changes in the MEP amplitude and rmsEMG (normalized to Mwave) give access to the excitability of the entire corticospinal pathway (above the neuromuscular junction) including the motor cortical and spinal motoneurones (Gandevia et al. 1999; Taylor et al. 2002). Thus, our data does not specifically determine whether the depression in the central excitability was due to a reduction in the responsiveness of the motor cortical neurons, the spinal motoneurone and/or the 
316 corticospinal transmission. Given that we did not find any alteration in the duration of the SP, it

317 could be inferred that the reduction in the central excitability following ROLLING could not be 318 due to a GABAnergic intracortical inhibition. Further studies are required to quantify the effect 319 of rolling massage on the acute and chronic adaptations of the cortical and spinal segments of the 320 central nervous system.

Investigating the influence of rolling massage on maximal force output was not the main purpose of the present study, as our previous experiments had demonstrated that the technique did not alter the maximal force generating capacity (Sullivan et al. 2013; Halperin et al. 2014;

324 Cavanaugh et al. 2017). In line with our previous findings, the MVC force output did not show any significant change following ROLLING. The data suggest that, although rolling massage can modulate the corticospinal excitability responses, it does not cause any change in the maximal 327 force out. frequency of ROLLING over both VL and VM muscles, it is not clear why the MEP and rmsEMG recorded from the VM did not show similar results to VL. A plausible explanation for 331 different responses of VM and VL might be that the VL is the primary knee extensor during low 332 intensity isometric knee extensions (Zhang et al. 2003). Therefore, our data suggest that different 333 segments of quadriceps muscle may demonstrate various responses to ROLLING depending on 334 the background contraction intensity.

A methodological consideration for the current study is that a 24 to 48 hours interval was assigned between the two intervention sessions. Although there is no documented research that has explored the potential long-term adaptation of corticomotor responses following rolling massage, our cross-over study design warrants further considerations. In addition, the current 
339 study does not directly evaluate the influence of ROLLING on activation of muscle spindles and

340 group III and IV afferent receptors located in the quadriceps muscle. Thus, further studies with

341 more sophisticated neurophysiological measurements of afferent and efferent reflexive pathways

342 are required to elucidate the influence of rolling massage on neuromuscular performance. could modulate the responsiveness of corticospinal circuitries innervating the knee extensor

346 muscles. However, the observed effects were highly dependent on the background knee

347 extension voluntary contractions during which the neuromuscular measurements were recorded.

Acknowledgements. The MITACS accelerate grant financially supported this study. We would

349 like to acknowledge the contributions of Dr. Thamir Alkanani for his organization and

350 preparation of the laboratory and equipment.

351 Conflict of interest. The authors report no conflicts of interest associated with this manuscript. 


\section{REFERENCE}

Aboodarda, S.J., Spence, A.J., and Button, D.C. 2015. Pain pressure threshold of a muscle tender spot increases following local and non-local rolling massage. BMC musculoskeletal disorders. $16(1): 265$.

Beardsley, C., and Škarabot, J. 2015. Effects of self-myofascial release: A systematic review. J. Bodyw. Mov. Ther. 19(4): 747-58.

Behara, B., and Jacobson, BH. 2017. Acute Effects of Deep Tissue Foam Rolling and Dynamic Stretching on Muscular Strength, Power, and Flexibility in Division I Linemen. J. Strength. Cond. Res. 31(4): 888-892.

Behm, D.G., Peach, A., Maddigan, M., Aboodarda, S.J., DiSanto, M.C., Button, D.C., and Maffiuletti, N.A. 2013. Massage and stretching reduce spinal reflex excitability without affecting twitch contractile properties. J. Electromyogr. Kinesiol. 23(5): 1215-21.

Bradbury-Squires, D.J., Noftall, J.C., Sullivan, K.M., Behm, D.G., Power, K.E., and Button D,C. 2015. Roller-massager application to the quadriceps and knee-joint range of motion and neuromuscular efficiency during a lunge. J. Athl. Train. 50(2): 133-40.

Button, D.C., and Behm, D.G. 2008. The effect of stimulus anticipation on the interpolated twitch technique. J. Sports. Sci. Med. 7(4): 520-4.

Canadian Society for Exercise Physiology. 2011. Physical Activity Readiness QuestionnairePlus. http://www.csep.ca/CMFiles/publications/parq/PARQPlusforCEPs_12Sept2011.pdf.

Carson, R.G., Riek, S., and Bawa, P. 1999. Electromyographic activity, H-reflex modulation and corticospinal input to forearm motoneurones during active and passive rhythmic movements. Human Mov. Sci. 18(2-3): 307-343

Cavanaugh, M.T., Döweling, A., Young, J.D., Quigley, P.J., Hodgson, D.D., Whitten, J.H., Reid, J.C., Aboodarda, S.J., and Behm, D.G. 2017. An acute session of roller massage prolongs voluntary torque development and diminishes evoked pain. Eur. J. Appl. Physiol. 117(1): 109117.

Cohen, J. 1988. Statistical power analysis for the behavioral sciences. 2nd edn. Erlbaum, Hillsdale.

Dishman, J.D., and Bulbulian R. 2001. Comparison of effects of spinal manipulation and massage on motoneuron excitability. Electromyogr. Clin. Neurophysiol. 41(2): 97-106.

Fourment, A., Chennevelle, J.M., Belhaj-Saif, A., and Maton, B. 1996. Responses of motor cortical cells to short trains of vibration. Exp. Brain Res. 111: 208-14. 
Gandevia, S.C., Petersen, N., Butler, J.E., and Taylor, J.L. 1999. Impaired response of human motoneurones to corticospinal stimulation after voluntary exercise. J. Appl. Physiol. 15:749-59.

Goldberg, J., Sullivan, S.J., and Seaborne, D.E. 1992. The effect of two intensities of massage on H-reflex amplitude. Phys. Ther. 72(6): 449-57.

Halperin, I., Aboodarda, S.J., Button, D.C., Andersen, L.L., and Behm, D.G. 2014. Roller massager improves range of motion of plantar flexor muscles without subsequent decreases in force parameters. Int. J. Sports Phys. Ther. 9(1): 92-102.

Hermens, H.J., Freriks, B., Merletti, R., Ha“gg, G.G., Stegeman, D., Blok, J., Rau, G., Disselhorst-Klug, C. 1999. SENIAM 8: European recommendations for surface Electromyography, deliverable of the SENIAM project. Roessingh Research and Development.

Kaelin-Lang, A.1., Luft, A.R., Sawaki, L., Burstein, A.H., Sohn, Y.H., Cohen, L.G. 2002. Modulation of human corticomotor excitability by somatosensory input. J. Physiol. 540(Pt 2): $623-33$.

Kossev, A., Siggelkow, S., Schubert, M., Wohlfarth, K., Dengler, R. 1999. Muscle vibration: different effects on transcranial magnetic and electrical stimulation. Muscle Nerve, 22(7): 946-8.

Kovaleski, J.E., Heitman, R.J., Gurchiek, L.R., Trundle, T.L. 1999. Reliability and effects of arm dominance on upper extremity isokinetic force, work, and power using the closed chain rider system. J. Athl. Train. 34(4): 358-61.

MacDonald, G.Z., Penney, M.D., Mullaley, M.E., Cuconato, A.L., Drak,e C.D., Behm, D.G., and Button, D.C. 2013. An acute bout of self-myofascial release increases range of motion without a subsequent decrease in muscle activation or force. J. Strength Cond. Res. 27: 812-821.

Morelli, M., Seaborne, D.E., and Sullivan, S.J. 1991. H-reflex modulation during manual muscle massage of human triceps surae. Arch. Phys Med. Rehabil. 72(11): 915-9.

Okamoto, T., Masuhara, M., and Ikuta, K. 2014. Acute effects of self-myofascial release using a foam roller on arterial function. J. Strength Cond. Res. 28(1): 69-73.

Pearcey, G.E., Bradbury-Squires, D.J., Kawamoto, J.E., Drinkwater, E.J., Behm, D.G., and Button, D.C. 2015. Foam rolling for delayed-onset muscle soreness and recovery of dynamic performance measures. J. Athl. Train. 50(1): 5-13.

Ridding, M.C. and Taylor, J.L. 2001. Mechanisms of motor-evoked potential facilitation following prolonged dual peripheral and central stimulation in humans. J. Physiol. 537(Pt 2): $623-31$.

Rossi, S., Hallett, M., Rossini, P.M., Pascual-Leone, A. 2011. Screening questionnaire before TMS: an update. Clin. Neurophysiol. 122(8): 1686. 
Schleip, R. 2003a. Fascial plasticity - a new neurobiological explanation: Part 1. J. Bodywork Mov. Ther. 7(1): 11-19.

Schleip, R. 2003b. Fascial plasticity - a new neurobiological explanation: Part 2. J. Bodywork Mov. Ther. 7(2): 104-116.

Schnitzler, A. and Benecke R. 1994. The silent period after transcranial magnetic stimulation is of exclusive cortical origin: evidence from isolated cortical ischemic lesions in man. Neurosci. Lett. 180(1): 41-5.

Siggelkow, S., Kossev, A., Schubert, M., Kappels, H.H., Wolf, W., and Dengler, R. 1999. Modulation of motor evoked potentials by muscle vibration: the role of vibration frequency. Muscle and Nerve, 22(11): 1544-8.

Smith, L., and Brouwer, B. 2005. Effectiveness of muscle vibration in modulating corticospinal excitability. J. Rehabil. Res. Dev. 42(6): 787-94.

Souron, R., Farabet A., Féasson. L., Belli, A., Millet, G.Y., Lapole, T. 2017.

Eight weeks of local vibration training increases dorsiflexor muscles cortical voluntaryactivation. J. Appl. Physiol. doi: 10.1152/japplphysiol.00793.2016. [Epub ahead of print]

Steyvers, M., Levin, O., Verschueren, S.M., and Swinnen, S.P. 2003. Frequency-dependent effects of muscle tendon vibration on corticospinal excitability: a TMS study. Exp. Brain Res. 151(1): 9-14.

Sullivan, S.J., and Williams, L.R., Seaborne DE, Morelli M. 1991. Effects of massage on alpha motoneuron excitability. Phys. Ther. 71(8): 555-60.

Sullivan, S.J., Seguin, S., Seaborne, D., and Goldberg, J. 1993. Reduction of H-reflex amplitude during the application of effleurage to the triceps surae in neurologically healthy subjects. Physiotherapy Theory and Praclice, 9, 25-31.

Sullivan, K.M., Silvey, D.B., Button, D.C., and Behm, DG. 2013. Roller-Massager application to the hamstrings increases sit-and reach range of motion within five to ten seconds without performance impairments. Int. J. Sports. Phys. Ther. 8: 228-236.

Taylor, J.L., Petersen, N.T., Butler, J.E., and Gandevia, S.C. 2002. Interaction of transcranial magnetic stimulation and electrical transmastoid stimulation in human subjects. J. Physiol. 15: 949-58.

Zhang, L.Q., Wang, G., Nuber, G.W., Press, J.M., and Koh, JL. 2003. In vivo load sharing among the quadriceps components. J. Orthop. Res. 21(3): 565-71. 


\section{TABLES}

Table 1. The absolute values for the neurophysiological parameters recorded from knee extenosrs (VL and VM) at $10 \%$ of MVC at the baseline and following the four sets of the two interventions (CONTROL and ROLLING).

\begin{tabular}{|c|c|c|c|c|c|c|}
\hline $\mathbf{V L}$ & & Baseline & Set 1 & Set 2 & Set 3 & Set 4 \\
\hline \multirow[t]{2}{*}{ MEP/Mmax * } & CONTROL & $.27(.09)$ & $.28(.13)$ & $.27(.08)$ & $.28(.14)$ & $.28(.08)$ \\
\hline & ROLLING & $.33(.12)$ & $.34(.18)$ & $.30(.15)$ & $.30(.12)$ & $.29(.12)$ \\
\hline \multirow[t]{2}{*}{ rmsEMG/Mmax * } & CONTROL & $\begin{array}{c}.0062 \\
(.0028)\end{array}$ & $\begin{array}{c}.0061 \\
(.0028)\end{array}$ & $\begin{array}{c}.0063 \\
(.0029)\end{array}$ & $\begin{array}{l}.0060 \\
(.0029)\end{array}$ & $\begin{array}{c}.0062 \\
(.0030)\end{array}$ \\
\hline & ROLLING & $\begin{array}{l}.0067 \\
(.0023)\end{array}$ & $\begin{array}{c}.0059 \\
(.0018)\end{array}$ & $\begin{array}{c}.0056 \\
(.0015)\end{array}$ & $\begin{array}{c}.0058 \\
(.0017)\end{array}$ & $\begin{array}{c}.0057 \\
(.0021)\end{array}$ \\
\hline \multirow[t]{2}{*}{ SP (ms) } & CONTROL & $167.3(82.2)$ & $172.8(84.7)$ & $174.9(85.2)$ & $169.2(83.3)$ & $170.1(84.4)$ \\
\hline & ROLLING & $169.4(81.3)$ & $177.4(75.2)$ & $169.1(77.1)$ & $173.8(78.9)$ & $180.6(86.2)$ \\
\hline VM & & Baseline & Set 1 & Set 2 & Set 3 & Set 4 \\
\hline \multirow[t]{2}{*}{ MEP/Mmax } & CONTROL & $.35(.25)$ & $.38(.28)$ & $.33(.18)$ & $.32(.24)$ & $.36(.19)$ \\
\hline & ROLLING & $.44(.20)$ & $.50(.33)$ & $.41(.20)$ & $.47(.19)$ & $.45(.25)$ \\
\hline \multirow[t]{2}{*}{ rmsEMG/Mmax } & CONTROL & $\begin{array}{l}.0045 \\
(.0016)\end{array}$ & $\begin{array}{l}.0046 \\
(.0016)\end{array}$ & $\begin{array}{l}.0047 \\
(.0017)\end{array}$ & $\begin{array}{l}.0045 \\
(.0014) \\
\end{array}$ & $\begin{array}{l}.0049 \\
(.0012)\end{array}$ \\
\hline & ROLLING & $\begin{array}{l}.0060 \\
(.0028)\end{array}$ & $\begin{array}{c}.0059 \\
(.0028)\end{array}$ & $\begin{array}{c}.0054 \\
(.0025) \\
\end{array}$ & $\begin{array}{c}.0058 \\
(.0032) \\
\end{array}$ & $\begin{array}{c}.0057 \\
(.0029) \\
\end{array}$ \\
\hline \multirow[t]{3}{*}{ SP (ms) } & CONTROL & $177.4(84.6)$ & $185.3(83.9)$ & $183.1(82.4)$ & $179.6(84.9)$ & $179.3(89.7)$ \\
\hline & ROLLING & $173.5(77.3)$ & $177.8(81.7)$ & $177.5(82.8)$ & $177.8(82.4)$ & $183.2(81.5)$ \\
\hline & & Baseline & - & - & - & $\begin{array}{c}\text { Post- } \\
\text { intervention }\end{array}$ \\
\hline \multirow[t]{2}{*}{ MVC force $(\mathrm{N})$} & CONTROL & $\begin{array}{c}659.0 \\
(134.6)\end{array}$ & 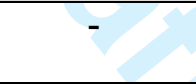 & & - & $\begin{array}{c}667.8 \\
(148.2)\end{array}$ \\
\hline & ROLLING & $\begin{array}{l}602.5 \\
(68.6) \\
\end{array}$ & - & - & - & $\begin{array}{c}603.6 \\
(122.2) \\
\end{array}$ \\
\hline
\end{tabular}

Note. MEP: motor evoked potential; Mmax: maximal compound muscle action potential; rmsEMG: root mean square of electromyographic activity; SP: silent period; VL: vastus lateralis and VM: vastus medialis; MVC: maximal voluntary knee extensions. $*$ denotes a significant condition effect $(\mathrm{p}<.05)$. 
13 Table 2. The absolute values for the neurophysiological parameters recorded from knee extenosrs (VL and VM) at

$1450 \%$ Of MVC at the baseline and following the four sets of the two interventions (CONTROL and ROLLING).

\begin{tabular}{|c|c|c|c|c|c|c|}
\hline VL & & Baseline & Set 1 & Set 2 & Set 3 & Set 4 \\
\hline \multirow[t]{2}{*}{ MEP/Mmax } & CONTROL & $.80(.19)$ & $.80(.20)$ & $.77(.21)$ & $.79(.26)$ & $.77(.28)$ \\
\hline & ROLLING & $.72(.16)$ & $.70(.19)$ & $.65(.17)$ & $.73(.20)$ & $.66(.22)$ \\
\hline \multirow[t]{2}{*}{ rmsEMG/Mmax } & CONTROL & $.029(.015)$ & $.026(.014)$ & $.028(.015)$ & $.028(.014)$ & $.029(.016)$ \\
\hline & ROLLING & $.030(.009)$ & $.032(.010)$ & $.030(.006)$ & $.032(.011)$ & $.031(.009)$ \\
\hline \multirow[t]{2}{*}{$\mathrm{SP}(\mathrm{ms})$} & CONTROL & $121.2(29.1)$ & $120.4(29.3)$ & $123.8(35.2)$ & $119.9(30.1)$ & $120.5(33.9)$ \\
\hline & ROLLING & $111.3(22.1)$ & $110.3(18.9)$ & $112.4(81.9)$ & $112.2(22.4)$ & $106.4(24.1)$ \\
\hline VM & & Baseline & Set 1 & Set 2 & Set 3 & Set 4 \\
\hline \multirow[t]{2}{*}{ MEP/Mmax } & CONTROL & $.73(.18)$ & $.72(.24)$ & $.72(.20)$ & $.72(.20)$ & $.68(.18)$ \\
\hline & ROLLING & $.64(.16)$ & $.59(.16)$ & $.59(.11)$ & $.62(.17)$ & $.57(.14)$ \\
\hline \multirow[t]{2}{*}{ rmsEMG/Mmax } & CONTROL & $.032(.015)$ & $.029(.014)$ & $.031(.017)$ & $.031(.018)$ & $.031(.017)$ \\
\hline & ROLLING & $.027(.009)$ & $.029(.010)$ & $.025(.008)$ & $.028(.009)$ & $.029(.011)$ \\
\hline \multirow[t]{3}{*}{$\mathrm{SP}(\mathrm{ms})$} & CONTROL & $118.4(29.6)$ & $115.8(29.9)$ & $120.4(36.9)$ & $117.8(31.6)$ & $116.2(32.0)$ \\
\hline & ROLLING & $111.9(26.3)$ & $109.1(19.8)$ & $109.3(22.9)$ & $109.4(22.0)$ & $105.6(25.2)$ \\
\hline & & Baseline & - & - & - & $\begin{array}{c}\text { Post- } \\
\text { intervention }\end{array}$ \\
\hline \multirow[t]{2}{*}{ MVC force $(\mathrm{N})$} & CONTROL & $\begin{array}{r}695.6 \\
(111.6) \\
\end{array}$ & - & - & - & $\begin{array}{l}737.9 \\
(97.0) \\
\end{array}$ \\
\hline & ROLLING & $\begin{array}{c}750.4 \\
(129.8) \\
\end{array}$ & - & - & - & $\begin{array}{c}725.3 \\
(108.4) \\
\end{array}$ \\
\hline
\end{tabular}

15

16

17

18

19

20

Note. MEP: motor evoked potential; Mmax: maximal compound muscle action potential; rmsEMG: root mean square of electromyographic activity; SP: silent period; VL: vastus lateralis and VM: vastus medialis; MVC: maximal voluntary knee extensions. 
Figure 1. Representative traces from a single subject for the MEPs and Mmax recorded from VL at 10\% of MVC at the baseline and following each set of intervention (CONTROL and ROLLING). MEP: motor evoked potentials; Mmax: compound muscle action potential.

Figure 2. The mean and SD of MEPs (normalized to Mwave) recorded from VL at $10 \%$ (panel A) and $50 \%$ MVCs (panel B). ${ }^{*}$ denotes a significantly lower value $(P=0.046)$ during the ROLLING compared to the CONTROL session.

Figure 3. The mean and SD of rmsEMG (normalized to Mwave) recorded from VL at $10 \%$ (panel A) and $50 \%$ MVCs (panel B). * denotes a significantly lower value $(P=0.041)$ following the ROLLING compared to the CONTROL session. 
FIGURE 1

$\begin{array}{llllll}\text { Baseline } 1 & \text { Baseline } 2 & \text { Set } 1 & \text { Set } 2 & \text { Set } 3 & \text { Set } 4\end{array}$
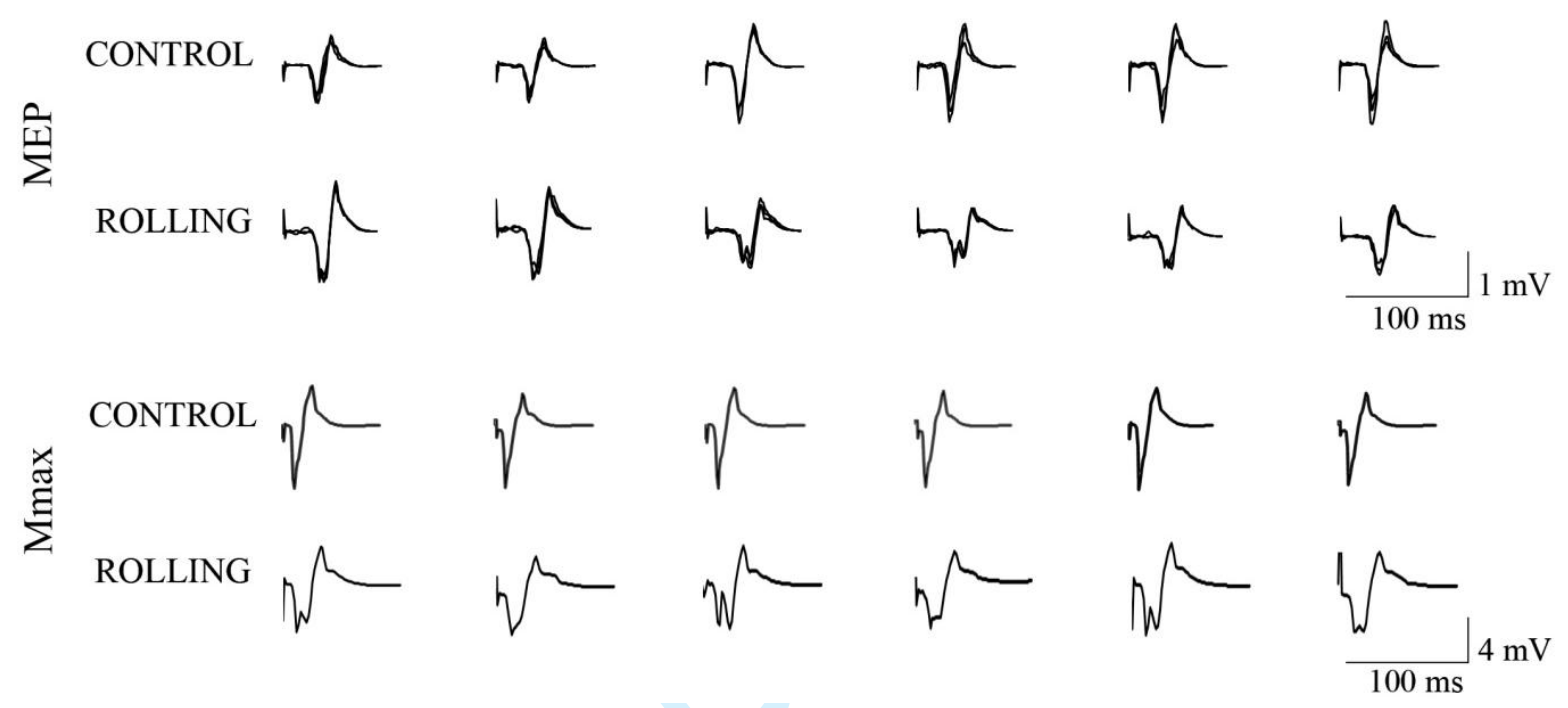

5

6

7

8

9

10 
FIGURE 2

A

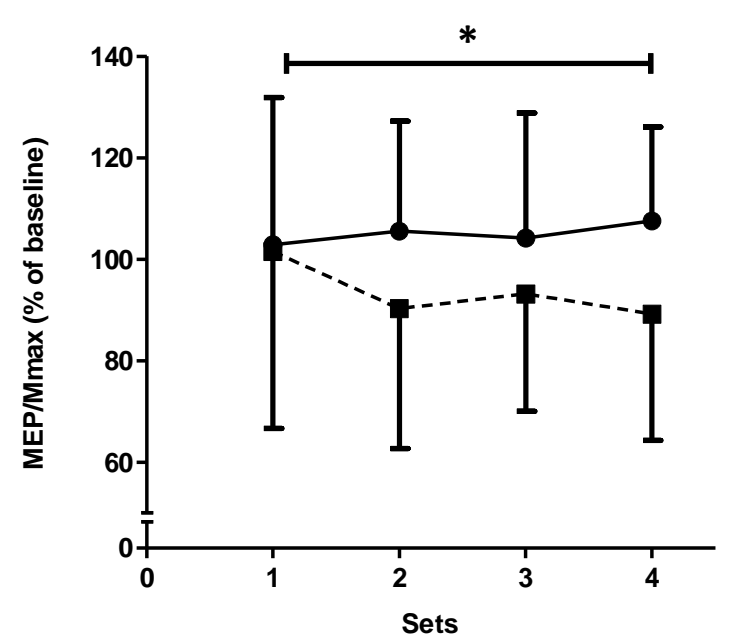

B

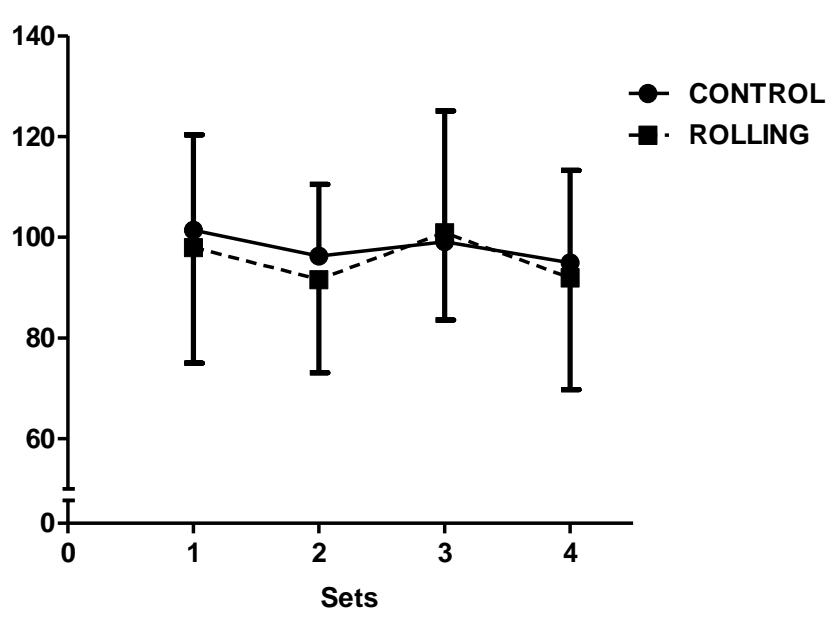

6
7 
FIGURE 3

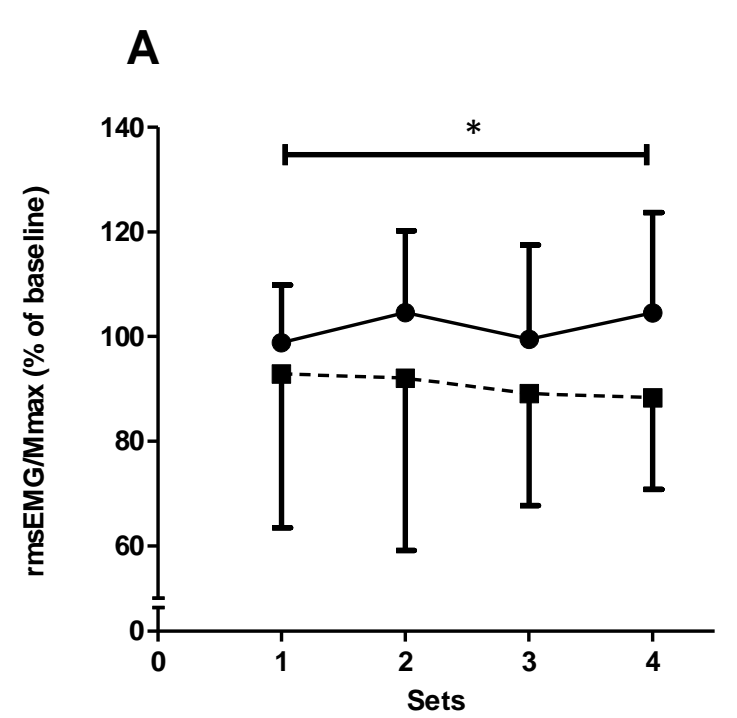

\section{B}

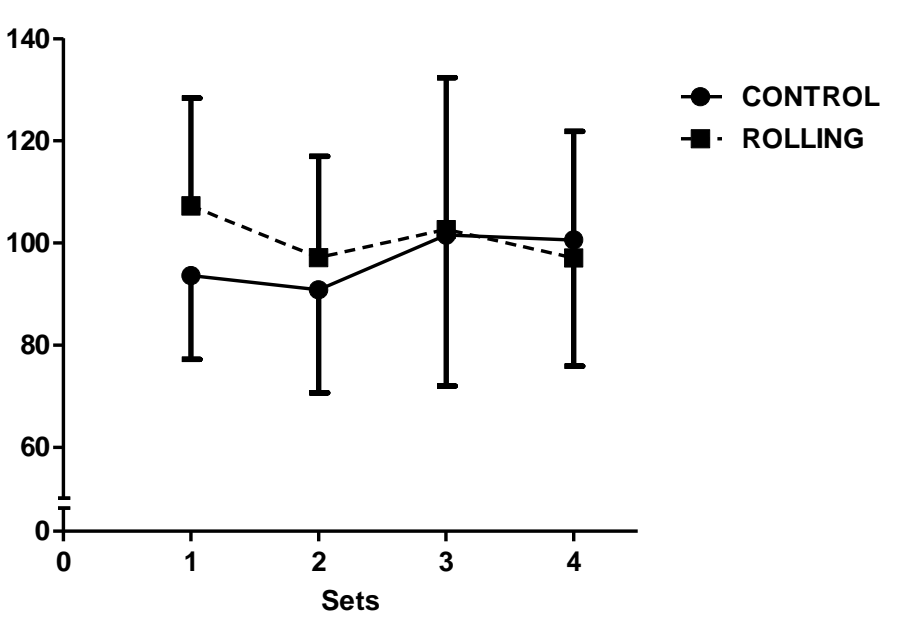

